\title{
Prediction of depression and anxiety via patient-assessed tremor severity, not physician-reported motor symptom severity, in patients with Parkinson's disease or essential tremor who have undergone deep brain stimulation
}

\author{
Rebecca L. Achey, BS, ${ }^{1}$ Erin Yamamoto, BS, ${ }^{1}$ Daniel Sexton, BS, ${ }^{1}$ Christine Hammer, MD, ${ }^{2}$ \\ Bryan S. Lee, MD, ${ }^{3}$ Robert S. Butler, MS, ${ }^{4}$ Nicolas R. Thompson, MS, ${ }^{4}$ Sean J. Nagel, MD, ${ }^{5}$ \\ Andre G. Machado, MD, PhD,, ${ }^{5,6}$ and Darlene A. Lobel, MD ${ }^{5,6}$ \\ ${ }^{1}$ Cleveland Clinic Lerner College of Medicine of Case Western Reserve University; ${ }^{3}$ Department of Neurosurgery, Neurological \\ Institute; ${ }^{5}$ Center for Neurological Restoration, Department of Neurosurgery, Neurological Institute; ${ }^{4}$ Department of Quantitative \\ Health Science, Cleveland Clinic; ${ }^{6}$ Department of Biomedical Engineering, Case Western Reserve University, Cleveland, \\ Ohio; and 'Department of Neurological Surgery, Thomas Jefferson University and Jefferson Hospital for Neuroscience, \\ Philadelphia, Pennsylvania
}

OBJECTIVE Deep brain stimulation (DBS) is an effective therapy for movement disorders such as idiopathic Parkinson's disease (PD) and essential tremor (ET). However, some patients who demonstrate benefit on objective motor function tests do not experience postoperative improvement in depression or anxiety, 2 important components of quality of life (QOL). Thus, to examine other possible explanations for the lack of a post-DBS correlation between improved objective motor function and decreased depression or anxiety, the authors investigated whether patient perceptions of motor symptom severity might contribute to disease-associated depression and anxiety.

METHODS The authors performed a retrospective chart review of PD and ET patients who had undergone DBS at the Cleveland Clinic in the period from 2009 to 2013. Patient demographics, diagnosis (PD, ET), motor symptom severity, and QOL measures (Primary Care Evaluation of Mental Disorders 9-item Patient Health Questionnaire [PHQ-9] for depression, Generalized Anxiety Disorder 7-item Scale [GAD-7], and patient-assessed tremor scores) were collected at 4 time points: preoperatively, postoperatively, 1-year follow-up, and 2-year follow-up. Multivariable prediction models with solutions for fixed effects were constructed to assess the correlation of predictor variables with PHQ-9 and GAD-7 scores. Predictor variables included age, sex, visit time, diagnosis (PD vs ET), patient-assessed tremor, physicianreported tremor, Unified Parkinson's Disease Rating Scale part III (UPDRS-III) score, and patient-assessed tremor over time.

RESULTS Seventy PD patients and 17 ET patients were included in this analysis. Mean postoperative and 1-year follow-up UPDRS-III and physician-reported tremor scores were significantly decreased compared with preoperative scores $(p<0.0001)$. Two-year follow-up physician-reported tremor was also significantly decreased from preoperative scores $(p<0.0001)$. Only a diagnosis of PD $(p=0.0047)$ and the patient-assessed tremor rating $(p<0.0001)$ were significantly predictive of depression. A greater time since surgery, in general, significantly decreased anxiety scores $(p<$ $0.0001)$ except when a worsening of patient-assessed tremor was reported over the same time period $(p<0.0013)$.

CONCLUSIONS Patient-assessed tremor severity alone was predictive of depression in PD and ET following DBS. This finding suggests that a patient's perception of illness plays a greater role in depression than objective physical disability regardless of the time since surgical intervention. In addition, while anxiety may be attenuated by DBS, patient-assessed return of tremor over time can increase anxiety, highlighting the importance of long-term follow-up for behavioral health

\footnotetext{
ABBREVIATIONS ADL = activity of daily living; DBS = deep brain stimulation; DSM-IV = Diagnostic and Statistical Manual of Mental Disorders, 4th Edition; ET = essential tremor; FTM = Fahn-Tolosa-Marin tremor rating scale; GAD-7 = Generalized Anxiety Disorder 7-item Scale; PD = Parkinson's disease; PHQ-9 = 9-item Patient Health Questionnaire; QOL = quality of life; UPDRS-III = Unified Parkinson's Disease Rating Scale part III.
}

SUBMITTED January 4, 2017. ACCEPTED August 28, 2017.

INCLUDE WHEN CITING Published online February 23, 2018; DOI: 10.3171/2017.8.JNS1733. 


\begin{abstract}
features in chronic neurological disorders. Together, these data suggest that the patient experience of motor symptoms plays a role in depression and anxiety - a finding that warrants consideration when evaluating, treating, and following movement disorder patients who are candidates for DBS.
\end{abstract}

https://thejns.org/doi/abs/10.3171/2017.8.JNS1733

KEY WORDS patient-perceived tremor; depression; anxiety; deep brain stimulation; quality of life; Parkinson's disease; essential tremor; functional neurosurgery

$\mathrm{D}$ EEP brain stimulation (DBS) is an effective treatment for motor symptoms in movement disorders such as idiopathic Parkinson's disease (PD) and essential tremor (ET). ${ }^{1,8,39}$ Patients with PD classically present with bradykinesia, rigidity, and resting tremor, as well as nonmotor symptoms, such as mood changes and cognitive decline. In contrast, those with ET present with action tremors, such as postural tremor (stabilizing the hands) or kinetic tremor (coordinated movement of the hands). ${ }^{7}$ Despite differences in presentation, it is increasingly recognized that both PD and ET have nonmotor features, including cognitive and psychiatric disturbance (depression, anxiety, and apathy). ${ }^{13,20,21}$ However, it is not definitively established whether objective motor function improvement following DBS plays a role in alleviating depression and/or anxiety in movement disorder patients. Furthermore, it is not known whether the impact of DBS on depression or anxiety differs depending on the divergent pathophysiological mechanisms responsible for tremor in PD versus ET.

Several randomized controlled trials have demonstrated improvements in quality of life (QOL) measures after DBS device implantation in patients with movement disorders. ${ }^{8,13,39}$ These improvements include a reduction in depression and anxiety. ${ }^{13,42}$ However, many patients do not experience these QOL improvements following DBS surgery. ${ }^{6,32}$ In fact, one prospective analysis found that, on average, patient-assessed depression and anxiety levels were unchanged 8 years post-DBS surgery. ${ }^{12}$ Improvement in QOL after DBS surgery has been explained by studies demonstrating an inverse correlation between increased QOL scores on the 39-item Parkinson's Disease Questionnaire (PDQ-39) and the time that patients spend in the off condition when symptoms are poorly controlled. ${ }^{6,31,33}$ This suggests that a patient's perception of their motor symptom severity, as well as the degree to which activities of daily living (ADLs) are impaired, can contribute to overall patient QOL self-assessment.

Importantly, though PD patients suffer from myriad motor symptoms, studies demonstrate that tremor can play a significant role in their depression, anxiety, and self-esteem.22 In fact, studies have shown that DBS for tremor in both PD and ET can improve the performance of ADLs. ${ }^{26,27}$ Thus, given the evidence for tremor's effect on mood and DBS-related tremor improvement in the performance of ADLs, we reasoned that the perception of tremor's effect on ADLs following surgery might have some relationship with postoperative depression and anxiety. Given the contradictory literature regarding depression or anxiety following DBS, the aim of this study was to elucidate the role of both objective physician-reported motor function and subjective patient-assessed motor func- tion in QOL outcomes after DBS surgery. Specifically, we hypothesized that improvement in physician-reported motor scores would correlate with decreased depression and anxiety given the well-established role of DBS in decreasing motor symptoms in PD and ET that impair daily functioning. Next, we hypothesized that patient-assessed motor symptom severity in both PD and ET would predict postoperative measures of depression and anxiety following DBS surgery given the possible role of patient perception in the experience of disease severity.

\section{Methods}

This study was approved by the Cleveland Clinic Foundation's institutional review board. We retrospectively identified patients diagnosed with PD and ET who had undergone DBS surgery at our institution in the period from January 2009 to January 2013, with 2-year follow-up visits into 2015. Patient demographics, diagnosis (PD, ET), motor symptom severity, and QOL measures (Primary Care Evaluation of Mental Disorders 9-item Patient Health Questionnaire [PHQ-9] for depression, Generalized Anxiety Disorder 7-item Scale [GAD-7], and patient-assessed tremor scores) were collected from the charts. Available motor scores and QOL reports were collected at 4 time points: preoperatively, 6-8 weeks postoperatively, 1-year follow-up, and 2-year follow-up. One- or 2-year followup appointment data were excluded if the appointment occurred more than 3 months from the planned interval follow-up date from surgery. Data were extracted from the electronic medical record and collected using Research Electronic Data Capture (REDCap), following institutional review board-approved methods for de-identification and protection of patient identifiers.

\section{Motor Symptom Severity Rating}

Data were collected on both physician-reported motor symptom severity during clinical encounters and patientassessed tremor severity-related functional impairment. Physician-reported symptom severity for PD patients was assessed using part III of the Movement Disorder Society's Unified Parkinson's Disease Rating Scale (UPDRS-III), a subset of the full UPDRS that specifically evaluates clinician-scored motor function. ${ }^{14,30}$ Physician-reported tremor scores were reported as the sum of individual tremor scores recorded for each body part in the UPDRS. Tremor scores for ET patients were collected by scoring physical examination tremor findings for each body part and for certain activities: at rest, straight arm postural, winged postural, finger to nose, and drinking from a cup. Scores for each body part and/or activity ranged from 0 (no tremor) to 4 (most severe) in accordance with the Fahn-Tolosa- 
TABLE 1. Patient characteristics in PD and ET

\begin{tabular}{lcccc}
\hline \multicolumn{1}{c}{ Parameter } & Total & PD & ET & p Value \\
\hline No. of patients & 87 & 70 & 17 & \\
\hline Mean age in yrs & $63.7 \pm 9.1$ & $62.7 \pm 9.0$ & $67.9 \pm 8.5$ & $0.036^{*}$ \\
\hline $\begin{array}{c}\text { Mean disease } \\
\text { duration in yrs }\end{array}$ & $10.9 \pm 8.0$ & $9.7 \pm 5.3$ & $16.2 \pm 14.0$ & $0.003^{*}$ \\
\hline Sex & & & \\
\hline M & $63(72.4)$ & $54(77.1)$ & $9(52.9)$ & \\
\hline F & $24(27.6)$ & $16(22.9)$ & $8(47.1)$ & \\
\hline Target type & & & & $<0.001 \S$ \\
\hline Unknown & $1(1.1)$ & $0(0.0)$ & $1(5.9)$ & \\
\hline STN & $69(79.3)$ & $69(98.6)$ & $0(0.0)$ & \\
\hline GPi & $1(1.1)$ & $1(1.4)$ & $0(0.0)$ & \\
\hline VIM & $16(18.4)$ & $0(0.0)$ & $17(100)$ & \\
\hline DBS type & & & & $<0.001 \ddagger$ \\
\hline DBS It & $44(50.6)$ & $28(40.0)$ & $16(94.1)$ & \\
\hline DBS rt & $18(20.7)$ & $17(24.3)$ & $1(5.9)$ & \\
\hline DBS bilat & $25(28.7)$ & $25(35.7)$ & $0(0.0)$ & \\
\hline
\end{tabular}

$\mathrm{GPi}=$ globus pallidus internus; STN = subthalamic nucleus; VIM = ventral intermediate nucleus.

Values expressed as the mean \pm standard deviation or number $(\%)$.

* ANOVA.

† Data not available for 3 patients.

$\ddagger$ Pearson's chi-square test.

$\S$ Fisher's exact test.

Marin tremor rating scale (FTM), a well-validated tremor scoring system. ${ }^{35}$ Tremor grades for the left and right side of the body were added for the composite tremor score.

Patient-assessed tremor scores were recorded using part C of the FTM before each visit. ${ }^{11}$ Specifically, the questionnaire asked patients to rate tremor-related impairment in 7 areas contributing to overall patient functionality during everyday activities: 1) speaking, 2) feeding, 3) bringing liquids to the mouth, 4) hygiene, 5) dressing, 6) writing, and 7) working. Impairment for each category was ranked on a scale from 0 (normal) to 4 (severely impaired). Twenty-eight was the highest achievable score, indicating maximum disability, whereas 0 was the lowest achievable score.

\section{Quality of Life Measures: PHQ-9 and GAD-7}

The PHQ-9 and GAD-7 are patient-assessed screening instruments that serve as validated standardized measures of QOL. ${ }^{18,23,40,41}$ The PHQ-9 includes 9 questions (3 points each) regarding symptoms that correlate with the Diagnostic and Statistical Manual of Mental Disorders, 4th Edition (DSM-IV) criteria for diagnosing major depression. A score of 10 is generally accepted as the cutoff to identify major depression, with scores of $0-4$ indicating minimal depression and scores of 5-9 denoting moderate depression. ${ }^{17}$ In the PD population, a score of 9 offers optimal sensitivity and specificity for screening for major depression. ${ }^{2}$ The GAD-7 is a 7-question screening tool to assess the frequency of anxiety symptoms over a 2 -week period. Question scores range from 0 (not at all) to 3 (nearly every day), in which scores $>5$ indicate mild anxiety, $>10$ indicate moderate anxiety, and $>15$ indicate severe anxiety. A cutoff score of 10 has $89 \%$ sensitivity and $82 \%$ specificity for identifying GAD in a primary care setting. ${ }^{29,34}$

\section{Statistical Methods}

The UPDRS-III and physician-reported tremor scores were obtained during the preoperative off-medication evaluation. Postoperative, 1-year follow-up, and 2-year follow-up UPDRS-III and physician-reported tremor scores were obtained during the on-medication/on-stimulation condition. Changes in the UPDRS-III scores in PD patients and physician-reported tremor scores in PD and ET patients were examined as a function of patient diagnosis (PD vs ET), age, and sex to identify possible significant differences. The UPDRS-III and physician-reported tremor scores were then compared across visits to test for significant pairwise mean differences between preoperative and subsequent measures. Dunnett's methods were used to adjust for multiple comparisons of means.

Multivariable repeated-measures regression models with solutions for fixed effects were constructed to assess the correlation of predictor variables with PHQ-9 and GAD-7 scores. An analysis of the colinearity of the variables of interest indicated that the predictor variables of patient diagnosis (PD vs ET), sex, age, visit time, patientassessed tremor, and physician-reported tremor, as well as the interaction between patient-assessed tremor scores and time, exhibited sufficient independence to permit their inclusion in a multivariable analysis. When the analysis was run using only PD patient data, the colinear assessment of the variables indicated that UPDRS-III scores could be added to the list of predictor variables. All analyses were also run with only bilaterally implanted DBS patients to examine the effect of persistent contralateral symptoms on QOL. Backward elimination regression methods were used to construct reduced models for all outcome variables of interest. Reduced models consist of only those variables that are statistically significantly correlated $(\mathrm{p}<$ 0.05 ) with PHQ-9 and GAD-7 scores. Patient demographics are reported as the mean \pm standard deviation. All other variables are reported as the mean \pm standard error. Statistical significance was set at $\mathrm{p}<0.05$.

\section{Results}

\section{Patient Demographics}

Eighty-seven patients were included in the analyses: 70 PD patients and 17 ET patients. They were $72.4 \%$ male (63 patients) and $27.6 \%$ female $(24 ; p<0.045)$. The PD patients were $77.1 \%$ male (54 patients) and $22.9 \%$ female (16), and all of them received subthalamic nucleus-targeted DBS (69 patients), with the exception of 1 patient who received globus pallidus internus-targeted DBS. Forty percent of the PD patients (28 patients) had left-sided DBS, whereas $35.7 \%$ (25) had bilateral DBS and 24.3\% (17) had rightsided DBS (Table 1). Essential tremor patients were 52.9\% male (9 patients) and $47.1 \%$ female (8). All ET patients underwent DBS targeting the ventral intermediate nucleus of the thalamus, and $94.1 \%$ of them (16 patients) received left-sided DBS and 5.9\% (1 patient) received right-sided 

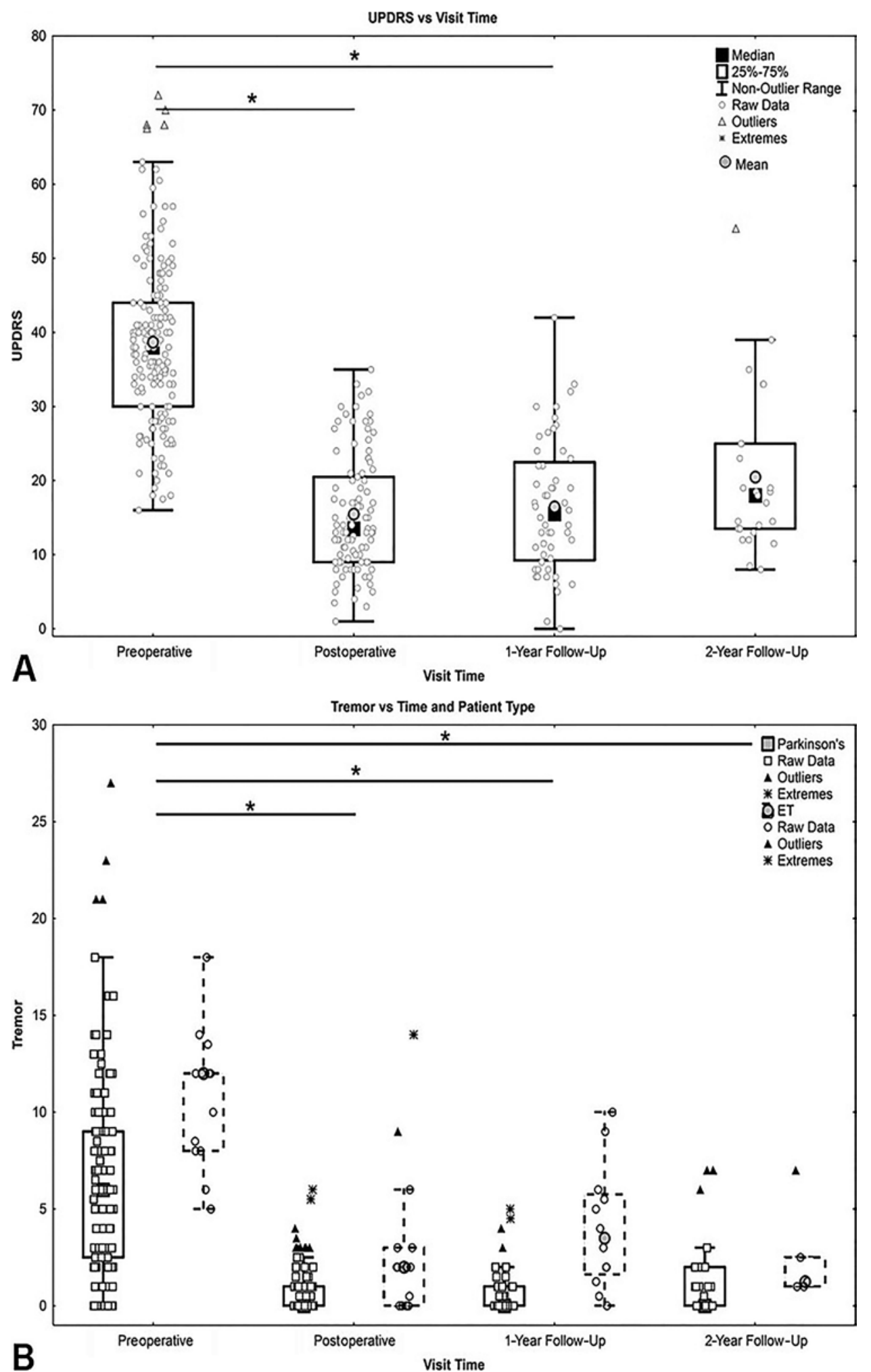

FIG. 1. A: The UPDRS motor examination (part III) scores (PD only) were collected at 4 different patient visits: 1) preoperatively, before DBS surgery; 2) postoperatively, within 6-8 weeks of DBS surgery; 3) 1-year follow-up; and 4) 2-year follow-up. There was a statistically significant decrease in the UPDRS-III scores following DBS at the postoperative visit and the 1-year follow-up visit. B: Physician-reported tremor scores for PD and ET patients were collected at all 4 time points. When controlling for time, ET patients had significantly higher physician-reported tremor scores than the PD patients. Additionally, there was a significant decrease in physician-reported tremor scores at all subsequent follow-up visits, compared to the preoperative scores. ${ }^{*} p<0.0001$. 


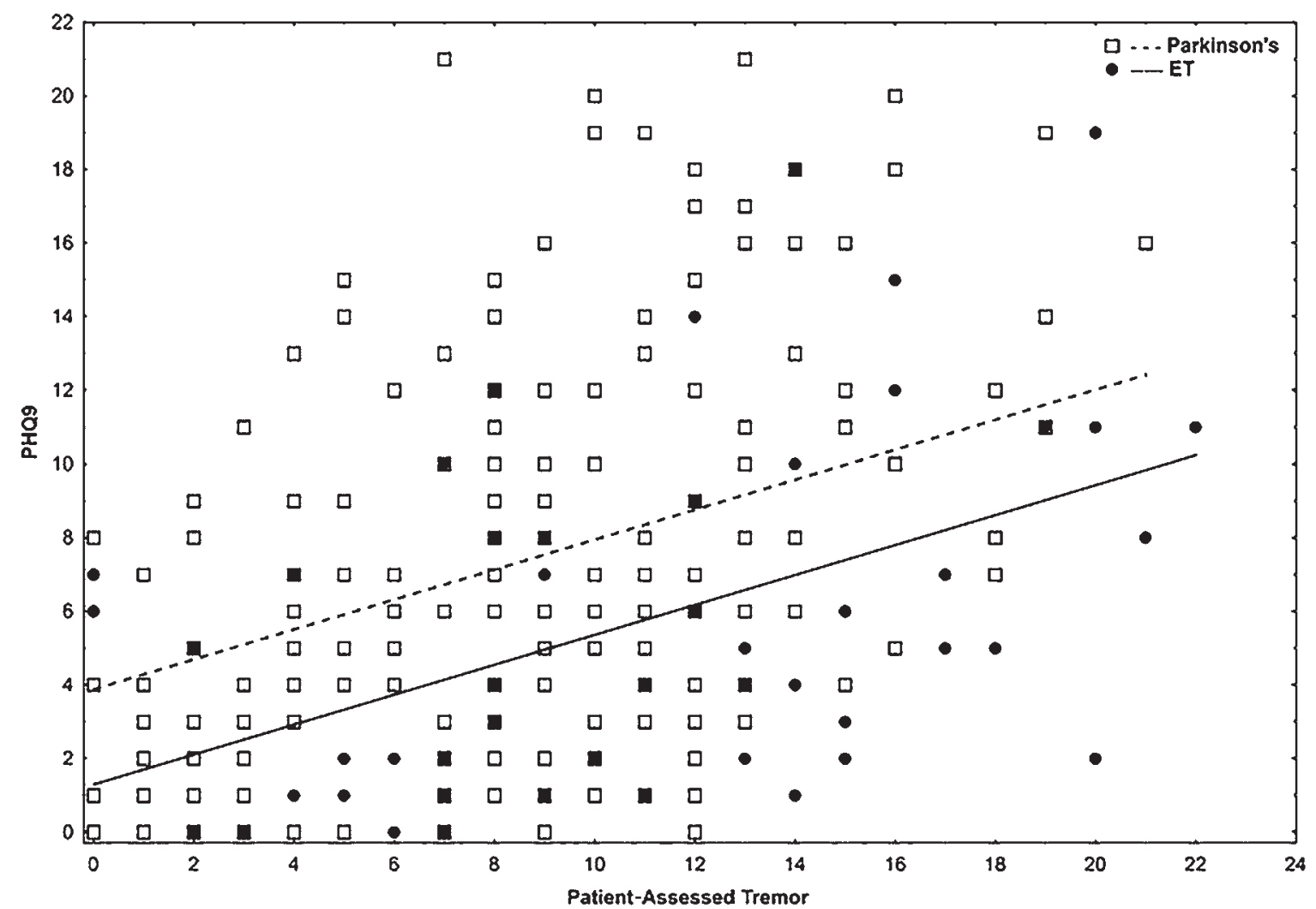

FIG. 2. The PHQ-9 scores were significantly associated with patient diagnosis $(p=0.0047)$ and patient-assessed tremor $(p<$ 0.0001 ), regardless of visit time. The dotted trajectory describes the association between patient-assessed tremor and PHQ-9 for PD patients, while the solid trajectory describes the association for ET patients. Patients with PD had higher PHQ-9 scores than the patients with ET $(p=0.0047)$. Patient-assessed tremor severity was positively correlated with PHQ-9 scores $(p<0.0001)$. The PHQ-9 scores were not associated with age, sex, or physician-reported tremor score. The equation describing the significant associations is PHQ-9 $=[1.29+2.58$ (disease type) +0.41 (patient-assessed tremor)], where PD gives disease type $=1$ and ET gives disease type $=0$ and patient-assessed tremor ranges from 0 to 22 , with 22 indicating greatest severity.

DBS. The mean age of the PD patients was $62.7 \pm 9.0$ years, which was significantly younger than the mean age of the ET patients $(67.9 \pm 8.5$ years; $\mathrm{p}=0.036)$. Disease duration was significantly shorter in the PD patients $(9.7$ \pm 5.3 years $)$ than in the ET patients $(16.2 \pm 14.0$ years; $p$ $=0.003)$.

\section{Physician-Reported Motor Severity Rating}

The mean UPDRS-III scores and their standard errors were as follows: preoperatively $38.75 \pm 0.80$, postoperatively $16.04 \pm 0.94$, 1-year follow-up $16.6 \pm 1.26$, and 2 -year follow-up $21.35 \pm 1.85$. Pairwise significant differences were observed for preoperative versus postoperative $(\mathrm{p}<0.0001)$ and preoperative versus 1-year follow-up $(\mathrm{p}<$ 0.0001 ; Fig. 1A).

Only patient diagnosis and visit time were significantly associated with changes in physician-reported tremor scores. When controlling for time, ET patients had significantly higher mean tremor scores $(5.58 \pm 0.62)$ than the PD patients $(2.23 \pm 0.28 ; \mathrm{p}<0.0001)$. There was a significant difference between preoperative mean physician-reported tremor scores $(8.18 \pm 0.38)$ and all subsequent mean measures at the postoperative follow-up $(2.45 \pm 0.41)$, 1-year follow-up (2.45 \pm 0.49$)$, and 2-year follow-up (2.54 \pm 0.66$)$, with $\mathrm{p}<0.0001$ for all comparisons (Fig. 1B).

\section{Quality of Life Measures: PHQ-9 and GAD-7}

After running backward elimination repeated-measures regression, we found that only patient diagnosis $(\mathrm{p}$ $=0.0047)$ and patient-assessed tremor $(p<0.0001)$ were significantly correlated with PHQ-9. Physician-reported tremor was not significantly correlated with PHQ-9. The prediction model equation is PHQ-9 $=[1.29+2.58$ (disease type) +0.41 (patient-assessed tremor)], where PD gives disease type $=1$ and ET gives disease type $=0$ and patientassessed tremor ranges from 0 to 22, with 22 indicating greatest severity (Fig. 2). The analysis was rerun for PD patients only with the UPDRS-III score included as an additional variable. After backward elimination regression, only patient-assessed tremor, and not physician-reported tremor or UPDRS-III score, significantly correlated with PHQ-9 ( $p<0.0001$; Fig. 3). The full equation for describing the relationship between PHQ-9 score and patient-assessed tremor in the PD-only cohort is PHQ-9 = [4.0421 +0.2861 (patient-assessed tremor)], where patient-assessed tremor ranges from 0 to 22 , with 22 indicating greatest severity. Sex, age, disease duration, visit time, physicianreported tremor, and UPDRS-III scores were not associated with depression. Similar results were obtained when performing the same analyses with bilateral DBS implantation patients only (data not shown). 


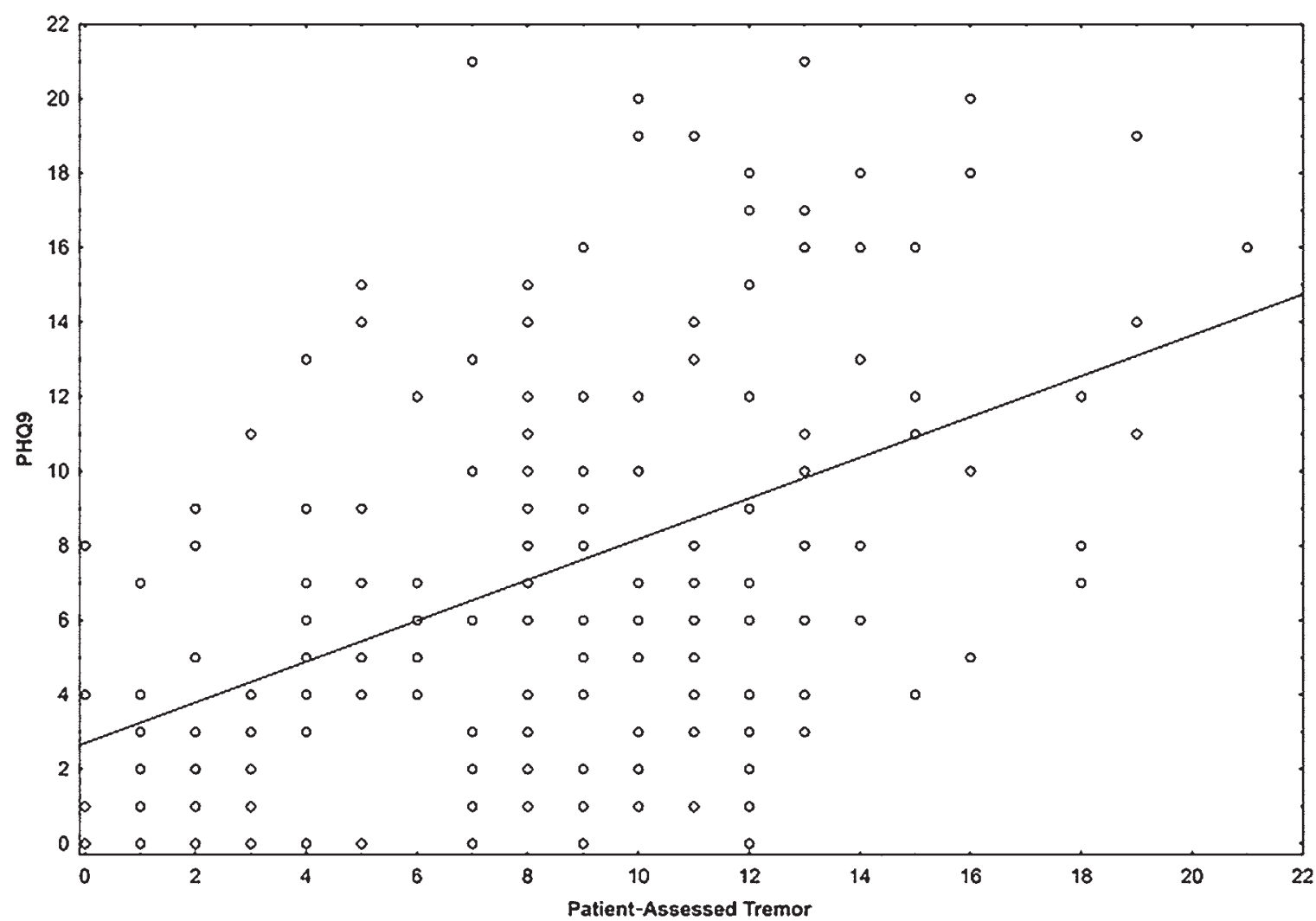

FIG. 3. In a PD-only cohort, PHQ-9 remained significantly associated with patient-assessed tremor $(p<0.0001)$, regardless of visit time. The PHQ-9 in the PD-only population was not associated with age, sex, physician-reported tremor, or UPDRS-III score. The equation describing the relationship between PHQ-9 score and patient-assessed tremor is PHQ-9 $=[4.0421+0.2861$ (patientassessed tremor)], where patient-assessed tremor ranges from 0 to 22, with 22 indicating greatest severity.

After running backward elimination repeated-measures regression, we found that only time $(\mathrm{p}<0.0001)$ and patient-assessed tremor over time $(\mathrm{p}<0.0013)$ were significantly correlated with the GAD-7 score. Physicianreported tremor was not significantly associated with the GAD-7 score. Time was negatively associated with GAD-7 scores, with each increment of time resulting in a decrease of 1.8 in the GAD-7 scores; however, a change in patientassessed tremor over time was positively correlated with GAD-7 scores ( $p<0.0013$; Fig. 4). The prediction model equation is GAD-7 $=[5.07088-1.8163$ (time interval) + 0.1067 (time interval)(patient-assessed tremor)], where time interval is defined as $0=$ preoperative, $1=$ postoperative, $2=1$-year follow-up, and 3 $=2$-year follow-up and patientassessed tremor varies from 0 to 22 , with 22 indicating greatest severity. When the analysis was rerun for PD patients only and the UPDRS-III scores were added to the possible correlated variables, the GAD-7 scores remained significantly associated only with time $(\mathrm{p}<0.0001)$ and patient-assessed tremor over time $(p=0.0014)$, and not physician-reported tremor or UPDRS-III scores (Fig. 5). The prediction model equation for PD patients only is GAD-7 = [5.96 - 1.95(time interval) + 0.1299(time interval)(patient-assessed tremor)]. Patient diagnosis, sex, age, physician-reported tremor, and UPDRS-III scores were not associated with anxiety. Similar results were obtained when performing the same analyses with bilateral DBS implantation patients only.

\section{Discussion}

The goal of this study was to investigate the effect of motor symptom severity on depression and anxiety in patients treated with DBS. We hypothesized that increased depression and anxiety, as measured by the PHQ-9 and GAD-7, would correlate with greater physician-reported and patient-assessed motor symptom severity, regardless of the disease entity. Furthermore, we anticipated that depression and anxiety would decrease over time in patients who experienced motor benefits following DBS surgery. Notably, we chose to analyze patient-assessed tremor and physician-reported tremor as potential predictors of QOL measures, independently from composite UPDRS scores, to distinguish between the impact of tremor and general PD symptomatology on QOL. To our knowledge, this is the first study on how the perception of tremor severity influences depression and anxiety in patients treated with DBS for either PD or ET.

Significant improvements in UPDRS-III and physicianreported tremor were present in PD and ET following DBS device implantation, thus confirming objective treatment efficacy in our patient population. However, we found that PHQ-9 scores were only positively correlated with patientperceived tremor severity $(\mathrm{p}<0.0001)$. Thus, in both PD and ET, greater patient-assessed tremor severity was predictive of higher depression scores regardless of time (preor post-DBS). Additionally, PD patients had significantly 


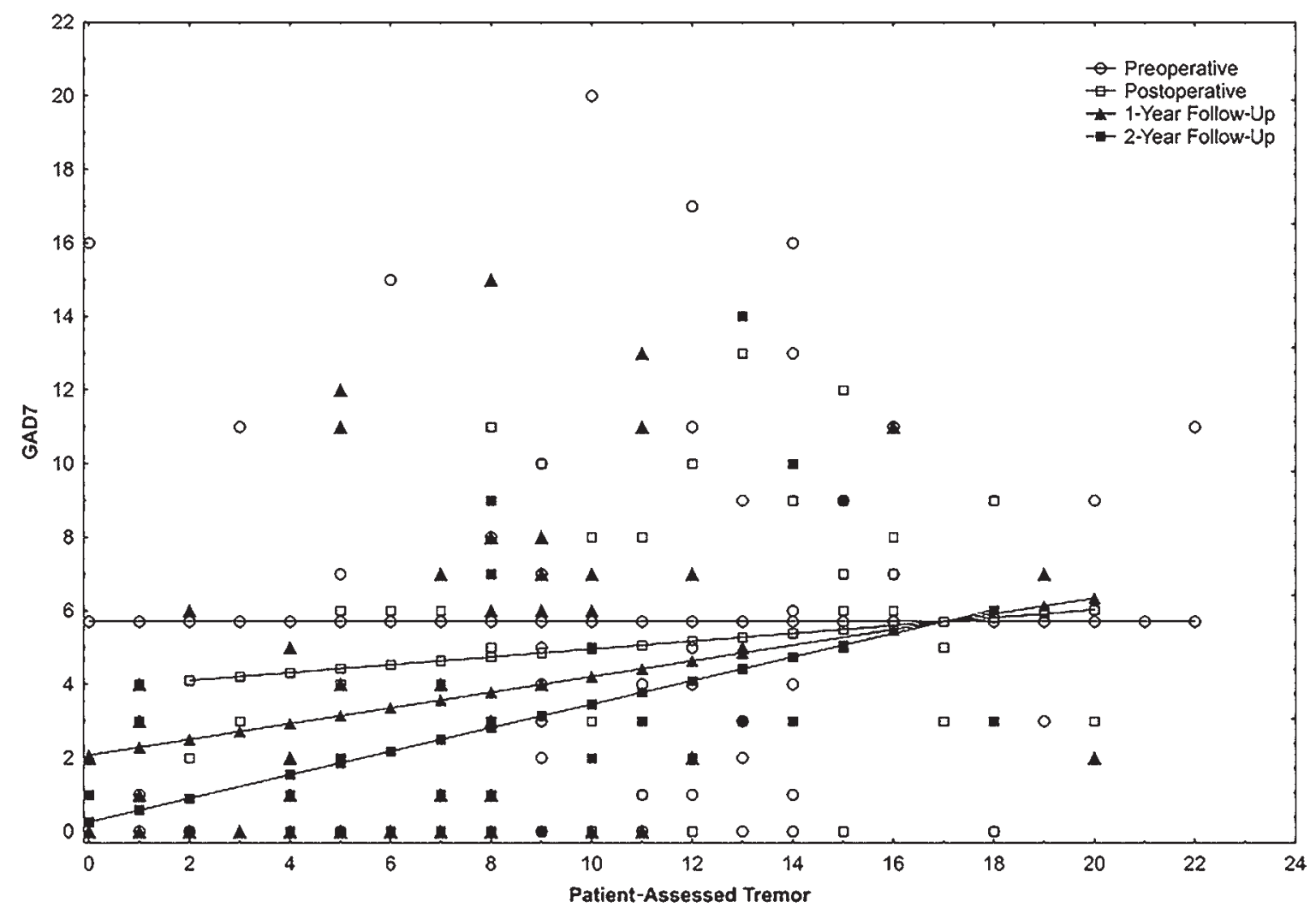

FIG. 4. The GAD-7 scores significantly decreased with time following surgery in both PD and ET cohorts $(p<0.0001)$. However, as the time from surgery increased, more severe patient-perceived tremor predicted higher GAD-7 scores $(p=0.0013)$. Unlike all postoperative visits, preoperative GAD-7 scores were not associated with patient-assessed tremor severity. There were no significant associations between GAD-7 scores and the other model variables, including age, sex, patient diagnosis, or physician-reported tremor scores. The equation describing the significant associations is GAD-7 $=[5.07088-1.8163$ (time interval) +0.1067 (time interval)(patient-assessed tremor)], where time interval is defined as $0=$ preoperative, $1=$ postoperative, $2=1$-year follow-up, and $3=2$-year follow-up and patient-assessed tremor varies from 0 to 22, with 22 indicating greatest severity.

higher PHQ-9 scores for any given self-assessed tremor severity than the ET patients. Unlike the depression models, GAD-7 scores were negatively correlated with time in both PD and ET in patients up to 2 years after surgery, with preoperative anxiety scores demonstrating no relation to patient-perceived tremor severity. However, as the time from surgery increased, patient-perceived worsening of tremor exacerbated anxiety. Interestingly, neither physician-reported tremor assessment for ET or PD nor composite UPDRS-III scores for PD significantly correlated with depression or anxiety.

Previously, studies have found an association between depression and objective measures of overall disease severity. $3,28,36,37$ For instance, one study of $32 \mathrm{PD}$ patients with depression had significantly higher scores on UPDRS-III than a matched cohort of PD patients without depression. ${ }^{28}$ Similarly, Stella et al. reported a significant positive association between depression and UPDRS scores (functional, motor, and total) in PD..$^{37}$ The same correlation has also been noted in ET, with one study reporting a significant association between tremor severity and depression scores. ${ }^{3}$ However, other studies have not detected an association between depression and functional scores. ${ }^{5,16}$ Our study demonstrates a novel finding in that patient assessment of tremor severity was the only predictor of the level of de- pression. This suggests that a physician's objective evaluation of motor symptom severity may not be congruent with a patient's experience of their symptoms. Furthermore, in light of the contradictory body of literature, in PD and ET, a patient's perception of their illness rather than their objective physical disability may play a greater role in the severity of their depression.

A retrospective study design is limited in its power to detect a causal link between depression and patient-perceived tremor severity. Thus, there are several possible explanations that warrant consideration. For example, patients with depression may have an inaccurate perception of the severity of their illness, resulting in the inability to identify objective physical improvement. Alternatively, depression may be a reaction to the severity of their experienced physical symptoms regardless of objective measurements; however, evidence for such "reactive depression" is lacking in the context of PD..$^{10}$ Another explanation could be related to unfulfilled expectations of DBS surgery. On the other hand, the physician's assessment of tremor severity may underestimate the true severity of a patient's motor symptoms in daily life given the focused nature of clinical encounters. Additionally, physician-reported tremor is based on a limited range of clinical findings, which may not fully capture the disability experienced by patients in 


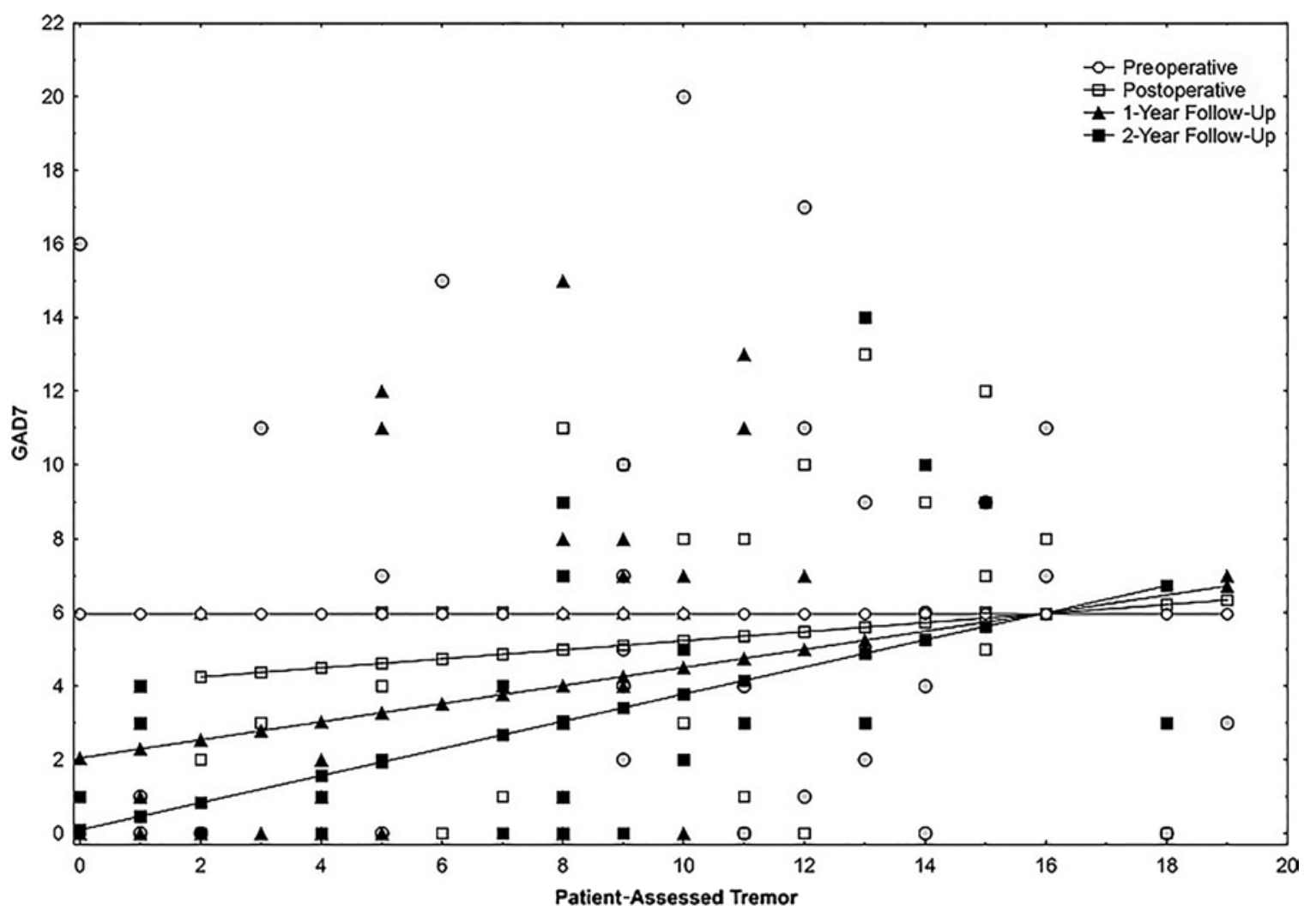

FIG. 5. In a PD-only cohort, the GAD-7 scores significantly decreased with time following surgery $(p<0.0001)$. However, as time from surgery increased, more severe patient-perceived tremor predicted higher GAD-7 scores $(p=0.0014)$. Unlike all postoperative visits, preoperative GAD-7 scores were not associated with patient-assessed tremor severity. The GAD-7 scores in the PD-only population were not associated with age, sex, physician-reported tremor, or UPDRS-III scores. The equation describing the associations between time and patient-assessed tremor over time is GAD-7 $=[5.96-1.95$ (time interval) +0.1299 (time interval) (patient-assessed tremor)].

their ADLs. Regardless of depression's cause, the ability of patient-assessed tremor to predict depression adds a new tool to gauge a patient's perception of how PD- and ET-related symptoms impact QOL. The patient-assessed tremor questionnaire could also serve as a novel, simple measure for identifying difficulty with ADLs, thus allowing for efficient referral to occupational therapy and psychiatric professionals to improve functional independence and enhance QOL following DBS surgery.

In our study, patients diagnosed with PD had higher depression scores for any given patient-perceived tremor severity score. This finding is in keeping with the literature suggesting that patients with PD have higher rates of depression than those with other chronic diseases. ${ }^{24}$ However, another study comparing depression in ET versus PD revealed no differences between the 2 cohorts. ${ }^{25}$ While it is possible that the PD patients in our cohort had greater depression than the ET patients, this finding could also be explained by recent evidence to suggest that ET patients with depression have decreased subjective experience of depression compared with controls. ${ }^{19}$ Notably, they experience less "reported sadness," anhedonia, and pessimistic thoughts but suffer more from impaired concentration. ${ }^{19}$ Thus, it is possible that the PHQ-9, which relies heavily on the DSM-IV criteria for major depression, underestimates the degree of depression in ET. Further investigations com- paring the experience of symptoms and depression in ET compared with PD may provide greater insight on this issue.

Although depression is one of the most common psychiatric comorbidities associated with chronic medical conditions, anxiety is also prevalent. ${ }^{4}$ However, our study found differing trends with regard to depression and anxiety, highlighting the necessity of considering each behavioral disorder as separate entities. In our study, anxiety decreased as a function of time following DBS surgery, consistent with prior studies in $\mathrm{PD}^{38}$ and ET. ${ }^{13}$ This points to tremor control as a significant contributor to a reduction in anxiety after DBS. However, patient-assessed tremor over time was positively correlated with GAD-7 scores. This implies that the decrease in anxiety due to DBS may be offset by a patient's perception of worsening tremor as the time from surgery elapses. This is supported by a study examining long-term follow-up after DBS, which revealed slight declines in emotional well-being and performance of ADLs, attributed to a wearing off of the "honeymoon effect" immediately following DBS surgery. ${ }^{9,13}$ There is also evidence from a study in ET that tremor control at 7 years post-DBS is not as effective as at 1 year. ${ }^{15}$ Thus, it becomes important to continue monitoring DBS patients' experiences of their disease and its treatment over the long term.

There are several strengths and limitations to this study. 
To our knowledge, this is the first study to examine the correlation between patient-perceived symptom control and depression or anxiety for up to 2 years postoperatively. Moreover, robust statistical modeling enabled us to verify the lack of colinearity between predictor variables and to use backward elimination regression to confirm statistically significant associations. Another study strength was the inclusion of both PD and ET patients to assess diseasespecific effects of motor symptom severity on depression and anxiety. However, ET-specific analyses were limited due to the low number of ET patients with complete data. This likely resulted from inconsistent patient participation in QOL form completion over the 2-year follow-up period and incomplete FTM motor exam scores at follow-up visits. As such, there is the possibility that the ET patients included here are not representative of the general ET population. Caution must therefore be exercised in interpreting the results of this study in ET specifically. Similarly, it must be noted that comorbid depression prior to surgery could impact patient-perceived motor dysfunction, just as unrealistic expectations of surgery could exacerbate psychological disturbance following surgical intervention, thus confounding the present study's findings. Additionally, the retrospective nature of this study prohibits us from concluding any causal relationship between patient-assessed tremor severity and depression or anxiety. This prevents us from entirely excluding additional factors contributing to post-DBS depression and anxiety, such as additional motor symptom progression, unrealistic expectations of surgery, or psychiatric comorbidity. Future investigations should employ a prospective study design with higher rates of ET patient enrollment to ensure adequate disease-specific analyses and greater control over confounding variables. Additionally, future studies should investigate the contribution of patient perception of the other cardinal motor symptoms in PD to QOL improvement following DBS.

\section{Conclusions}

In this study, in both PD and ET, greater patient-assessed tremor severity, but not physician-reported severity, was predictive of higher depression scores regardless of the time after DBS. The results indicate that a patient's perception of their illness rather than objective symptom severity assessment may play a greater role in associated behavioral disorders, regardless of DBS. Patient-assessed tremor may, therefore, provide a new tool for gauging how perceived symptoms impact QOL. In addition, while anxiety may be attenuated by DBS implantation, patient-perceived return of tremor symptoms following surgery may drive increased anxiety, highlighting the importance of long-term follow-up for behavioral disorders in surgically treated patients.

\section{References}

1. Baizabal-Carvallo JF, Kagnoff MN, Jimenez-Shahed J, Fekete R, Jankovic J: The safety and efficacy of thalamic deep brain stimulation in essential tremor: 10 years and beyond. J Neurol Neurosurg Psychiatry 85:567-572, 2014

2. Chagas MHN, Tumas V, Rodrigues GR, Machado-de-Sousa JP, Filho AS, Hallak JEC, et al: Validation and internal con- sistency of Patient Health Questionnaire-9 for major depression in Parkinson's disease. Age Ageing 42:645-649, 2013

3. Chandran V, Pal PK, Reddy JYC, Thennarasu K, Yadav R, Shivashankar N: Non-motor features in essential tremor. Acta Neurol Scand 125:332-337, 2012

4. Chen JJ, Marsh L: Anxiety in Parkinson's disease: identification and management. Ther Adv Neurol Disorder 7:52-59, 2014

5. Cole SA, Woodard JL, Juncos JL, Kogos JL, Youngstrom EA, Watts RL: Depression and disability in Parkinson's disease. J Neuropsychiatry Clin Neurosci 8:20-25, 1996

6. Daniels C, Krack P, Volkmann J, Raethjen J, Pinsker MO, Kloss M, et al: Is improvement in the quality of life after subthalamic nucleus stimulation in Parkinson's disease predictable? Mov Disord 26:2516-2521, 2011

7. Deuschl G, Elble R: Essential tremor-neurodegenerative or nondegenerative disease towards a working definition of ET. Mov Disord 24:2033-2041, 2009

8. Deuschl G, Schade-Brittinger C, Krack P, Volkmann J, Schäfer H, Bötzel K, et al: A randomized trial of deep-brain stimulation for Parkinson's disease. N Engl J Med 355:896908, 2006

9. Diamond A, Jankovic J: The effect of deep brain stimulation on quality of life in movement disorders. J Neurol Neurosurg Psychiatry 76:1188-1193, 2005

10. Edwards E, Kitt C, Oliver E, Finkelstein J, Wagster M, McDonald WM: Depression and Parkinson's disease: a new look at an old problem. Depress Anxiety 16:39-48, 2002

11. Fahn S, Tolosa E, Marin C: Clinical rating scale for tremor, in Jankovic J, Tolosa E (eds): Parkinson's Disease and Movement Disorders, ed 2. Baltimore: Williams \& Wilkins, 1993, pp 225-234

12. Fasano A, Romito LM, Daniele A, Piano C, Zinno M, Bentivoglio AR, et al: Motor and cognitive outcome in patients with Parkinson's disease 8 years after subthalamic implants. Brain 133:2664-2676, 2010

13. Fields JA, Tröster AI, Woods SP, Higginson CI, Wilkinson $\mathrm{SB}$, Lyons KE, et al: Neuropsychological and quality of life outcomes 12 months after unilateral thalamic stimulation for essential tremor. J Neurol Neurosurg Psychiatry 74:305311,2003

14. Goetz CG, Tilley BC, Shaftman SR, Stebbins GT, Fahn S, Martinez-Martin P, et al: Movement Disorder Societysponsored revision of the Unified Parkinson's Disease Rating Scale (MDS-UPDRS): scale presentation and clinimetric testing results. Mov Disord 23:2129-2170, 2008

15. Hariz GM, Blomstedt P, Koskinen LOD: Long-term effect of deep brain stimulation for essential tremor on activities of daily living and health-related quality of life. Acta Neurol Scand 118:387-394, 2008

16. Huber SJ, Paulson GW, Shuttleworth EC: Relationship of motor symptoms, intellectual impairment, and depression in Parkinson's disease. J Neurol Neurosurg Psychiatry 51:855-858, 1988

17. Kroenke K, Spitzer RL, Williams JBW, Löwe B: The Patient Health Questionnaire Somatic, Anxiety, and Depressive Symptom Scales: a systematic review. Gen Hosp Psychiatry 32:345-359, 2010

18. Kroenke K, Spitzer RL, Williams JBW: The PHQ-9: validity of a brief depression severity measure. J Gen Intern Med 16:606-613, 2001

19. Li ZW, Xie MJ, Tian DS, Li JJ, Zhang JP, Jiao L, et al: Characteristics of depressive symptoms in essential tremor. J Clin Neurosci 18:52-56, 2011

20. Lombardi WJ, Woolston DJ, Roberts JW, Gross RE: Cognitive deficits in patients with essential tremor. Neurology 57:785-790, 2001

21. Louis ED: Non-motor symptoms in essential tremor: A review of the current data and state of the field. Parkinsonism Relat Disord 22 (Suppl 1):S115-S118, 2016 
22. Louis ED, Machado DG: Tremor-related quality of life: A comparison of essential tremor vs. Parkinson's disease patients. Parkinsonism Relat Disord 21:729-735, 2015

23. Löwe B, Decker O, Müller S, Brähler E, Schellberg D, Herzog W, et al: Validation and standardization of the Generalized Anxiety Disorder Screener (GAD-7) in the general population. Med Care 46:266-274, 2008

24. Mayeux R: Behavioral manifestations of movement disorders. Parkinson's and Huntington's disease. Neurol Clin 2:527-540, 1984

25. Miller KM, Okun MS, Fernandez HF, Jacobson CE IV, Rodriguez RL, Bowers D: Depression symptoms in movement disorders: comparing Parkinson's disease, dystonia, and essential tremor. Mov Disord 22:666-672, 2007

26. Obwegeser AA, Uitti RJ, Witte RJ, Lucas JA, Turk MF, Wharen RE Jr: Quantitative and qualitative outcome measures after thalamic deep brain stimulation to treat disabling tremors. Neurosurgery 48:274-284, 2001

27. Ondo W, Jankovic J, Schwartz K, Almaguer M, Simpson RK: Unilateral thalamic deep brain stimulation for refractory essential tremor and Parkinson's disease tremor. Neurology 51:1063-1069, 1998

28. Papapetropoulos S, Ellul J, Argyriou AA, Chroni E, Lekka NP: The effect of depression on motor function and disease severity of Parkinson's disease. Clin Neurol Neurosurg 108:465-469, 2006

29. Plummer F, Manea L, Trepel D, McMillan D: Screening for anxiety disorders with the GAD-7 and GAD-2: a systematic review and diagnostic metaanalysis. Gen Hosp Psychiatry 39:24-31, 2016

30. Ramaker C, Marinus J, Stiggelbout AM, Van Hilten BJ: Systematic evaluation of rating scales for impairment and disability in Parkinson's disease. Mov Disord 17:867-876, 2002

31. Siderowf A, Jaggi JL, Xie SX, Loveland-Jones C, Leng L, Hurtig H, et al: Long-term effects of bilateral subthalamic nucleus stimulation on health-related quality of life in advanced Parkinson's disease. Mov Disord 21:746-753, 2006

32. Smeding HMM, Speelman JD, Huizenga HM, Schuurman PR, Schmand B: Predictors of cognitive and psychosocial outcome after STN DBS in Parkinson's Disease. J Neurol Neurosurg Psychiatry 82:754-760, 2011

33. Sobstyl M,Ząbek M, Górecki W, Mossakowski Z: Quality of life in advanced Parkinson's disease after bilateral subthalamic stimulation: 2 years follow-up study. Clin Neurol Neurosurg 124:161-165, 2014

34. Spitzer RL, Kroenke K, Williams JBW, Löwe B: A brief measure for assessing generalized anxiety disorder: the GAD-7. Arch Intern Med 166:1092-1097, 2006

35. Stacy MA, Elble RJ, Ondo WG, Wu SC, Hulihan J: Assessment of interrater and intrarater reliability of the FahnTolosa-Marin Tremor Rating Scale in essential tremor. Mov Disord 22:833-838, 2007

36. Starkstein SE, Mayberg HS, Leiguarda R, Preziosi TJ, Robinson RG: A prospective longitudinal study of depression, cognitive decline, and physical impairments in patients with Parkinson's disease. J Neurol Neurosurg Psychiatry 55:377-382, 1992
37. Stella F, Banzato CEM, Barasnevicius Quagliato EMA, Viana MA: Depression in patients with Parkinson's disease: impact on functioning. J Neurol Sci 272:158-163, 2008

38. Straits-Tröster K, Fields JA, Wilkinson SB, Pahwa R, Lyons KE, Koller WC, et al: Health-related quality of life in Parkinson's disease after pallidotomy and deep brain stimulation. Brain Cogn 42:399-416, 2000

39. Weaver FM, Follett K, Stern M, Hur K, Harris C, Marks WJ $\mathrm{Jr}$, et al: Bilateral deep brain stimulation vs best medical therapy for patients with advanced Parkinson disease: a randomized controlled trial. JAMA 301:63-73, 2009

40. Wild B, Eckl A, Herzog W, Niehoff D, Lechner S, Maatouk I, et al: Assessing generalized anxiety disorder in elderly people using the GAD-7 and GAD-2 scales: results of a validation study. Am J Geriatr Psychiatry 22:1029-1038, 2014

41. Williams JR, Hirsch ES, Anderson K, Bush AL, Goldstein SR, Grill S, et al: A comparison of nine scales to detect depression in Parkinson disease: which scale to use? Neurology 78:998-1006, 2012

42. Witt K, Daniels C, Reiff J, Krack P, Volkmann J, Pinsker MO, et al: Neuropsychological and psychiatric changes after deep brain stimulation for Parkinson's disease: a randomised, multicentre study. Lancet Neurol 7:605-614, 2008

\section{Disclosures}

Dr. Machado has been a consultant for St. Jude; holds a patent with Enspire, ATI, and Cardionomics; has received clinical or research support from Enspire and the NIH for the study described; and has received fellowship support from Medtronic.

\section{Author Contributions}

Conception and design: Achey, Lee. Acquisition of data: Achey, Yamamoto. Analysis and interpretation of data: Achey, Yamamoto, Thompson. Drafting the article: Lobel, Achey, Yamamoto, Sexton, Hammer, Lee. Critically revising the article: Lobel, Achey, Yamamoto, Sexton, Hammer, Lee, Butler, Nagel, Machado. Reviewed submitted version of manuscript: Lobel, Achey, Yamamoto, Sexton, Hammer, Lee, Butler, Nagel, Machado. Approved the final version of the manuscript on behalf of all authors: Lobel. Statistical analysis: Achey, Butler, Thompson. Study supervision: Lobel.

\section{Supplemental Information \\ Previous Presentations}

Portions of this work were presented in poster form as proceedings at the American Society for Stereotactic and Functional Neurosurgery Biennial Meeting held in June 2016 in Chicago, Illinois, and at the American Association of Neurological Surgeons Annual Meeting held in May 2015 in Washington, DC.

\section{Correspondence}

Darlene A. Lobel: Cleveland Clinic, Cleveland, OH. lobeld@ ccf.org. 